OPEN ACCESS

Edited by:

Shuo Wang,

Capital Medical University, China

Reviewed by:

Ewa Maria,

Polish Academy of Sciences, Poland

Christoph Reinhardt,

Johannes Gutenberg University

Mainz, Germany

*Correspondence:

Jian Xia

xjian1216@csu.edu.cn

tThese authors have contributed equally to this work and share first authorship

Received: 02 March 2021 Accepted: 25 June 2021 Published: 30 July 2021

Citation:

Yu F, Feng X, Li X, Luo Y, Wei M, Zhao $T$ and $X i a ~ J ~(2021)$ Gut-Derived Metabolite Phenylacetylg/utamine and

White Matter Hyperintensities in Patients With Acute Ischemic Stroke. Front. Aging Neurosci. 13:675158. doi: 10.3389/fnagi.2021.675158

\section{Gut-Derived Metabolite Phenylacetylglutamine and White Matter Hyperintensities in Patients With Acute Ischemic Stroke}

\author{
Fang $\mathrm{Yu}^{1+}$, Xianjing Feng ${ }^{1 \dagger}$, Xi $\mathrm{Li}^{1}$, Yunfang Luo ${ }^{1}$, Minping Wei ${ }^{1}$, Tingting Zhao ${ }^{1}$ and \\ Jian Xia ${ }^{1,2,3 *}$ \\ ${ }^{1}$ Department of Neurology, Xiangya Hospital, Central South University, Changsha, China, ${ }^{2}$ Clinical Research Center for \\ Cerebrovascular Disease of Hunan Province, Central South University, Changsha, China, ${ }^{3}$ National Clinical Research Center \\ for Geriatric Disorders, Xiangya Hospital, Central South University, Changsha, China
}

Background: White matter hyperintensity $(\mathrm{WMH})$ burden is associated with a higher risk of ischemic stroke. Phenylacetylglutamine (PAGIn) is a gut microbiota-derived metabolite that may induce cardiovascular events by activating platelets and increasing the risk of thrombosis. The relationship between plasma PAGIn and WMH burden in patients with ischemic stroke is unknown. This study was designed to investigate the association between plasma PAGIn and WMH burden in patients with acute ischemic stroke.

Methods: A total of 595 patients with acute ischemic stroke were enrolled in this study within 14 days of symptom onset. The burden of WMH was evaluated using the Fazekas scale based on the fluid-attenuated inversion recovery sequence. The severity of overall WMH was defined as none-mild WMH (total Fazekas score 0-2) or moderate-severe $\mathrm{WMH}$ (total Fazekas score 3-6). Based on the severity of periventricular WMH (P-WMH) and deep WMH (D-WMH), patients were categorized into either a none-mild (Fazekas score 0-1) group or a moderate-severe (Fazekas score 2-3) group. Plasma PAGln levels were quantified using liquid chromatography-mass spectrometry.

Results: We found that patients with moderate-severe overall WMH showed higher plasma PAGIn levels than patients with none-mild overall WMH, and similar results were found in the analyses according to $\mathrm{P}-\mathrm{WMH}$ and $\mathrm{D}-\mathrm{WMH}$. The logistic regression analysis showed that the fourth PAGln quartile was independently associated with moderate-severe overall WMH (adjusted 95\% Cl 1.134-4.018) and P-WMH (adjusted 95\% Cl 1.174-4.226).

Conclusion: These findings suggest that higher plasma PAGIn levels are associated with moderate-severe overall $\mathrm{WMH}$ and $\mathrm{P}-\mathrm{WMH}$ in patients with acute ischemic stroke.

Keywords: metabolomics, phenylacetylglutamine, white matter hyperintensities, ischemic stroke, biomarkers 


\section{INTRODUCTION}

Stroke is a major cause of disability and death in China (Wu et al., 2019). White matter hyperintensity (WMH) is the most common radiological marker of small vessel disease (SVD) (Joutel and Chabriat, 2017), and mounting evidence has shown that WMH burden is related to the risk of first stroke, recurrent stroke, and poorer outcomes after stroke (Arsava et al., 2009; Park et al., 2019). Age and hypertension are widely considered to be the main risk factors for WMH (Rist et al., 2019), but they do not account for all the pathophysiological mechanisms of WMH. Therefore, identifying novel risk factors is crucial to improve our understanding of the etiology and consequences of WMH in patients with ischemic stroke.

Recently, altered circulating metabolites have been identified as contributing factors in stroke and cerebral small vessel disease (CSVD) (Nie et al., 2018; Janes et al., 2019). For instance, asymmetric dimethylarginine (ADMA) levels were found to be positively correlated with WMH burden in young asymptomatic patients (Janes et al., 2019). Phenylacetylglutamine (PAGln), a gut microbiota-derived metabolite, has been associated with adverse cardiovascular events, such as coronary artery disease and stroke (Nemet et al., 2020). PAGln is formed by the conjugation of glutamine and phenylacetate, which is derived from bacterial phenylalanine metabolism (Moldave and Meister, 1957). Higher plasma PAGln levels increase the risk of cardiovascular events which may be due to enhanced platelet activation and thrombosis potential (Nemet et al., 2020).

However, the relationship between circulating PAGln and WMH burden in ischemic stroke patients is unknown. Therefore, to enhance our knowledge of the predictive role of PAGln in WMH impairment, we prospectively investigated the relationship between circulating PAGln and $\mathrm{WMH}$ impairment in patients with ischemic stroke. This study represents the first cross-sectional study examining whether plasma PAGln levels are associated with WMH burden in ischemic stroke patients.

\section{MATERIALS AND METHODS}

\section{Study Participants}

This study included consecutive patients with ischemic stroke confirmed between August 2017 and October 2020. We recruited 595 patients with ischemic stroke confirmed by diffusionweighted imaging of the brain within 14 days of symptom onset. The other inclusion criterion was age $\geq 18$ years. We excluded patients with disabilities (Modified Rankin Scale score $\geq 2$ ) before stroke onset and those without fluid-attenuated inversion recovery sequence (FLAIR). This study was approved by the Ethics Committee of Xiangya Hospital. All participants provided written informed consent.

\section{Clinical Assessments}

We assessed demographic characteristics and medical history, including age, sex, vascular risk factors [i.e., hypertension, diabetes mellitus, dyslipidemia, coronary heart disease (CAD), smoking, and drinking], based on the definitions previously described in detail (Feng et al., 2021). Complete blood count, liver and kidney function, blood glucose, homocysteine, and serum lipids were determined from overnight fasting venous blood samples from each participant on the second day of admission.

\section{Fluid-Attenuated Inversion Recovery Sequence Magnetic Resonance Imaging Assessment of WMH}

Periventricular WMH (P-WMH) and deep WMH (D-WMH) were assessed on FLAIR images using the Fazekas scale, which ranges from 0 to 3 . We categorized the severity of $\mathrm{P}-\mathrm{WMH}$ and D-WMH as none-mild (Fazekas score $0-1$ ) or moderate-severe (Fazekas score 2-3) (Yu et al., 2018). The total Fazekas score was classified based on the sum of P-WMH and D-WMH (range 0-6). The severity of overall WMH was identified as follows: nonemild WMH (Fazekas score 0-2) or moderate-severe WMH (Fazekas score 3-6) (Zhu et al., 2020).

\section{Quantification of PAGIn}

Overnight fasting venous blood samples were collected as soon as possible on the second day of admission. The whole blood sample was centrifuged into plasma and stored at $-80^{\circ} \mathrm{C}$ until analysis. Plasma PAGln was quantified on an AB SCIEX TripleTOF 6500 system (AB SCIEX, Foster City, CA, USA) using liquid chromatography-mass spectrometry with $\mathrm{D}_{5}$-PAGln (CDN Isotopes, Cat \# D-6900) as an internal standard. First, plasma was diluted 10 -fold with $\mathrm{ddH}_{2} \mathrm{O}$, then $2 \mu \mathrm{l}$ of 1 ppm D5PAGIn was added to $48 \mu \mathrm{l}$ of diluted plasma, and the mixture was diluted 4-fold with ice-cold methanol and vortexed for $1 \mathrm{~min}$. The supernatant was then centrifuged at $21,000 \times g$ at $4{ }^{\circ} \mathrm{C}$ for $15 \mathrm{~min}$ and transferred to a clean vial for testing. Finally, $1 \mu \mathrm{l}$ of the supernatant was injected into an Acquity UPLC BEH C18 column (Waters, Herts, UK) for analysis $(50 \times 2.1 \mathrm{~mm}$, $1.7 \mu \mathrm{m})$. The column temperature was $40^{\circ} \mathrm{C}$, and the flow rate was $0.3 \mathrm{ml} / \mathrm{min}$, with the mobile phase A containing $0.1 \%$ acetic acid in water and mobile phase B containing $0.1 \%$ acetic acid in water. We used known PAGln concentrations to establish a standard curve for the determination of PAGln concentrations. The PAGln concentration of the standard was $10 \mathrm{ng} / \mathrm{ml}$. The intra-day coefficients of variation were $0.80-1.39 \%$, and the interday coefficients of variation were $4.80-6.00 \%$.

\section{Statistical Analysis}

We used SPSS 22.0 (IBM Corp., Armonk, NY, USA) and GraphPad Prism 8 (GraphPad Software, San Diego, CA, USA) for the statistical analysis. The participants were dichotomized according to WMH burden into none-mild and moderatesevere groups using the Fazekas scores. In addition, participants were divided into four groups according to the quartiles of plasma PAGln concentrations. Categorical variables were described as proportions, and continuous variables were described as mean $\pm \mathrm{SD}$ or medians [interquartile range (IQR)]. Continuous variables were compared using an ANOVA, Kruskal-Wallis test, or Mann-Whitney $U$ test, as appropriate. Categorical variables were analyzed using the Pearson's $\chi^{2}$ test. We conducted a logistic regression analysis using the following three models: an unadjusted model, a model adjusted for age and sex, and a model adjusted for age, sex, and the variables showing 
TABLE 1 | Baseline characteristics of patients with ischemic stroke according to PAGln quartiles.

\begin{tabular}{|c|c|c|c|c|c|}
\hline Variables & $\begin{array}{l}\text { First quartile } \\
\qquad n=149\end{array}$ & $\begin{array}{l}\text { Second quartile } \\
\qquad n=149\end{array}$ & $\begin{array}{c}\text { Third quartile } \\
\qquad n=149\end{array}$ & $\begin{array}{l}\text { Fourth quartile } \\
\qquad n=148\end{array}$ & $P$ value \\
\hline Fazekas score & $2.0(1.5-4.0)$ & $3.0(2.0-4.0)$ & $2.0(2.0-4.0)$ & $4.0(2.0-5.0)$ & $<0.001$ \\
\hline Age (years) & $54(48-62)$ & $60(51-66)$ & $63(54-70)$ & $67(60-72)$ & $<0.001$ \\
\hline Sex (male, $N, \%)$ & $95(63.8 \%)$ & $106(71.1 \%)$ & $103(69.1 \%)$ & 99 (66.9\%) & 0.562 \\
\hline $\operatorname{HBP}(N, \%)$ & $93(62.4 \%)$ & $113(75.8 \%)$ & $99(66.4 \%)$ & $119(80.4 \%)$ & 0.002 \\
\hline $\mathrm{DM}(N, \%)$ & $26(17.4 \%)$ & $47(31.5 \%)$ & $45(30.2 \%)$ & $54(36.5 \%)$ & 0.003 \\
\hline Hyperlipidemia (N, \%) & $46(30.9 \%)$ & $42(28.2 \%)$ & $40(26.8 \%)$ & 45 (30.4\%) & 0.855 \\
\hline $\operatorname{CAD}(N, \%)$ & $19(12.8 \%)$ & 15 (10.1\%) & $29(19.5 \%)$ & $39(26.4 \%)$ & $<0.001$ \\
\hline Smoking $(N, \%)$ & $68(45.6 \%)$ & $81(54.4 \%)$ & $71(47.7 \%)$ & $64(43.2 \%)$ & 0.253 \\
\hline Drinking $(N, \%)$ & $48(32.2 \%)$ & $58(38.9 \%)$ & 59 (39.6\%) & $48(32.4 \%)$ & 0.372 \\
\hline $\mathrm{SBP}(\mathrm{mmHg})$ & $140.0(125.0-154.0)$ & $144.0(129.0-157.0)$ & $143.0(130.0-156.0)$ & $142.5(134.8-158.5)$ & 0.135 \\
\hline DBP (mmHg) & $84.0(74.0-93.0)$ & $82.0(74.0-92.0)$ & $82.0(74.0-90.0)$ & $81.0(72.0-91.2)$ & 0.632 \\
\hline $\mathrm{BMl}$ & $23.5(22.0-25.1)$ & 23.6 (21.9-25.9) & $22.9(21.5-25.7)$ & $23.0(21.0-25.7)$ & 0.678 \\
\hline White blood cell count $\left(\times 10^{9} / \mathrm{L}\right)$ & $6.2(5.2-7.9)$ & $6.7(5.7-8.2)$ & $6.5(5.5-8.1)$ & $7.0(5.8-8.1)$ & 0.064 \\
\hline Platelet $\left(\times 10^{9} / \mathrm{L}\right)$ & $208.0(164.0-251.0)$ & $209.0(167.0-240.0)$ & $196.0(162.0-246.0)$ & $203.5(167.8-235.0)$ & 0.615 \\
\hline $\mathrm{BUN}(\mathrm{mmol} / \mathrm{L})$ & $4.6(3.9-5.6)$ & $4.9(4.1-6.0)$ & $5.2(4.1-6.2)$ & $5.6(4.6-7.2)$ & $<0.001$ \\
\hline eGFR (ml/min/1.73 m²) & $89.4(76.7-102.6)$ & $86.6(74.6-96.3)$ & $81.5(69.7-92.5)$ & $72.7(57.8-89.1)$ & $<0.001$ \\
\hline Uric acid ( $\mu \mathrm{mol} / \mathrm{L})$ & $335.9(96.4)$ & $341.1(270.6-385.4)$ & $307.3(245.0-381.8)$ & $332.3(273.2-387.4)$ & 0.175 \\
\hline $\mathrm{TC}(\mathrm{mmol} / \mathrm{L})$ & $3.9(3.3-5.0)$ & $4.4(3.6-5.2)$ & $4.2(3.5-5.0)$ & $4.3(3.5-5.2)$ & 0.110 \\
\hline TG (mmol/L) & $1.5(1.0-2.2)$ & $1.6(1.1-2.3)$ & $1.5(1.1-2.0)$ & $1.5(1.2-2.3)$ & 0.525 \\
\hline $\mathrm{HDL}(\mathrm{mmol} / \mathrm{L})$ & $1.0(0.8-1.2)$ & $1.0(0.8-1.2)$ & $1.0(0.9-1.2)$ & $1.0(0.9-1.1)$ & 0.757 \\
\hline LDL (mmol/L) & $2.4(1.9-3.1)$ & $2.6(2.1-3.2)$ & $2.6(2.1-3.3)$ & $2.7(2.2-3.3)$ & 0.078 \\
\hline Fasting blood-glucose (mmol/L) & $5.4(4.8-6.3)$ & $5.8(5.0-7.4)$ & $5.9(5.1-7.7)$ & $5.7(5.1-8.1)$ & 0.065 \\
\hline $\mathrm{HbA1c}(\%)$ & $5.7(5.4-6.3)$ & $5.9(5.5-6.9)$ & $5.9(5.5-7.4)$ & $6.0(5.5-7.3)$ & 0.017 \\
\hline Homocysteine ( $\mu \mathrm{mol} / \mathrm{L})$ & $12.3(10.6-14.6)$ & $13.4(11.5-16.7)$ & $13.2(11.3-15.6)$ & 14.7 (11.9-19.3) & $<0.001$ \\
\hline
\end{tabular}

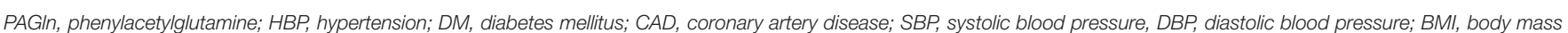

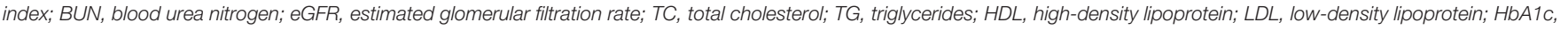
glycosylated hemoglobin A1c.

$P<0.05$ in the univariate analyses. We used the median to classify these confounding continuous variables in the regression analysis. Odds ratio (OR) and the 95\% CI were obtained. A Spearman rank correlation was used to identify the association between plasma PAGln levels and Fazekas scores. The value of PAGln for the prediction of WMH severity was evaluated using a receiver operating characteristics (ROC) curve, and the area under the ROC curve (AUC) was calculated. All tests were two-sided. Statistical significance was set at $P<0.05$.

\section{RESULTS}

\section{Clinical Characteristics of Patients With Ischemic Stroke}

A total of 595 patients (67.7\% male; median age, 61 years) with ischemic stroke were enrolled in our study. The median plasma PAGln level at admission was $2.06 \mu \mathrm{mol} / \mathrm{L}$. Quartiles of PAGln levels were as follows: first quartile, $<1.21 \mu \mathrm{mol} / \mathrm{L}$; second quartile, $1.21-2.06 \mu \mathrm{mol} / \mathrm{L}$; third quartile, $>2.06-3.34$ $\mu \mathrm{mol} / \mathrm{L}$; fourth quartile, $>3.34 \mu \mathrm{mol} / \mathrm{L}$. Higher PAGln quartiles were associated with high Fazekas scores, old age, high frequency of hypertension, diabetes mellitus, CAD, high levels of blood urea nitrogen and homocysteine, and low levels of estimated glomerular filtration rate (eGFR) (Table 1).

\section{The Association Between Plasma PAGIn and the Severity of Overall WMH According to Total Fazekas Scores}

There were 283 patients with none-mild overall WMH (total Fazekas score $0-2$ ) and 312 patients with moderate-severe overall WMH (total Fazekas score 3-6). When compared with patients with none-mild $\mathrm{WMH}$, patients with moderate-severe $\mathrm{WMH}$ were older and had a higher frequency of hypertension, diabetes mellitus, CAD, and higher levels of blood urea nitrogen and homocysteine. Lower levels of platelet count, eGFR, and total cholesterol were observed in moderate-severe WMH subjects (Table 2). We found higher plasma PAGln levels in patients with moderate-severe WMH than in patients with nonemild WMH [median 2.3 (IQR 1.5-3.8) vs. median 1.8 (IQR 1.0-2.8) $\mu \mathrm{mol} / \mathrm{L}, P<0.001$ ] (Figure 1A). Moreover, PAGln levels showed a limited correlation with the Fazekas score $(r=0.221, P<0.001)$ (Figure 2).

The results of the logistic regression analyses are shown in Table 3. In the unadjusted model, when using the first quartile as a reference, the second and fourth quartiles of PAGln levels 
TABLE 2 | Baseline characteristics of all patients according to the degree of overall WMH.

\begin{tabular}{|c|c|c|c|}
\hline Variables & $\begin{array}{c}\text { None-mild WMH } \\
\quad n=283\end{array}$ & $\begin{array}{l}\text { Moderate-severe WMH } \\
\qquad n=312\end{array}$ & $P$ value \\
\hline Age (years) & 55 (49-63) & $66(59-72)$ & $<0.001$ \\
\hline Sex (male, $N, \%$ ) & 200 (70.7\%) & 203 (65.1\%) & 0.144 \\
\hline $\operatorname{HBP}(N, \%)$ & $176(62.2 \%)$ & 248 (79.5\%) & $<0.001$ \\
\hline $\mathrm{DM}(N, \%)$ & $70(24.7 \%)$ & 102 (32.7\%) & 0.032 \\
\hline Hyperlipidemia (N, \%) & 83 (29.3\%) & 90 (28.8\%) & 0.897 \\
\hline CAD $(N, \%)$ & $33(11.7 \%)$ & $69(22.1 \%)$ & $<0.001$ \\
\hline Smoking (N, \%) & 143 (50.5\%) & $141(45.2 \%)$ & 0.193 \\
\hline Drinking $(N, \%)$ & 101 (35.7\%) & 112 (35.9\%) & 0.958 \\
\hline $\mathrm{SBP}(\mathrm{mmHg})$ & 142.0 (127.5-154.0) & 143.0 (130.0-159.2) & 0.086 \\
\hline DBP (mmHg) & 83.0 (74.0-93.0) & 82.0 (73.0-91.0) & 0.391 \\
\hline BMl & $23.4(22.0-25.2)$ & 23.3 (20.9-25.8) & 0.652 \\
\hline PAGln ( $\mu \mathrm{mol} / \mathrm{L})$ & $1.8(1.0-2.8)$ & $2.3(1.5-3.8)$ & $<0.001$ \\
\hline White blood cell count $\left(\times 10^{9} / \mathrm{L}\right)$ & $6.7(5.4-8.2)$ & $6.6(5.6-8.0)$ & 0.946 \\
\hline Platelet $\left(\times 10^{9} / \mathrm{L}\right)$ & 208.0 (171.0-250.5) & 199.0 (162.8-236.2) & 0.043 \\
\hline BUN (mmol/L) & $4.9(3.9-6.0)$ & $5.2(4.2-6.3)$ & 0.043 \\
\hline eGFR (ml/min/1.73 m²) & 88.5 (74.5-98.6) & 78.6 (64.5-90.2) & $<0.001$ \\
\hline Uric acid ( $\mu \mathrm{mol} / \mathrm{L})$ & 315.8 (272.9-380.1) & 331.4 (268.3-391.9) & 0.598 \\
\hline TC (mmol/L) & $4.2(3.4-5.2)$ & $4.2(3.5-4.9)$ & 0.234 \\
\hline TG (mmol/L) & $1.6(1.2-2.2)$ & $1.5(1.0-2.2)$ & 0.035 \\
\hline HDL (mmol/L) & $1.0(0.8-1.2)$ & $1.0(0.9-1.2)$ & 0.318 \\
\hline LDL (mmol/L) & $2.6(2.1-3.4)$ & $2.6(2.0-3.1)$ & 0.231 \\
\hline Fasting blood-glucose (mmol/L) & $5.6(5.0-7.1)$ & $5.6(5.0-7.7)$ & 0.702 \\
\hline $\mathrm{HbA1c}(\%)$ & $5.8(5.4-6.7)$ & $5.9(5.5-7.0)$ & 0.100 \\
\hline Homocysteine ( $\mu \mathrm{mol} / \mathrm{L})$ & $12.7(10.8-15.2)$ & $13.8(11.4-17.7)$ & 0.002 \\
\hline
\end{tabular}

WMH, white matter hyperintensity; HBP, hypertension; DM, diabetes mellitus; CAD, coronary artery disease; SBP, systolic blood pressure, DBP, diastolic blood pressure; BMI, body mass index; PAGIn, phenylacetylglutamine; BUN, blood urea nitrogen; eGFR, estimated glomerular filtration rate; TC, total cholesterol; TG, triglycerides; HDL, high-density lipoprotein; $L D L$, low-density lipoprotein; HbA1c, glycosylated hemoglobin A1c.

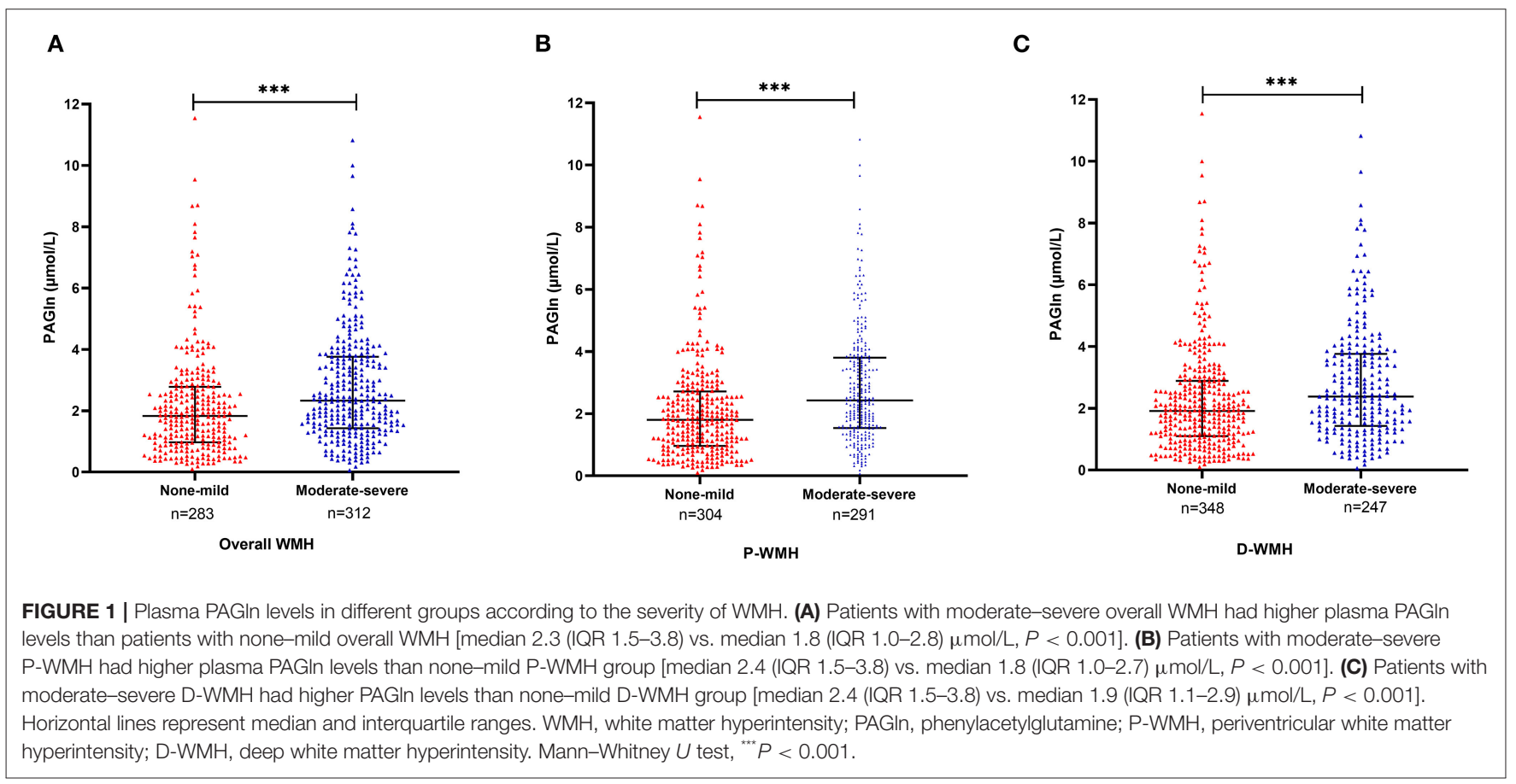


were independently associated with moderate-severe WMH (OR 2.212 and $95 \%$ CI $1.390-3.522$ for the second quartile and OR 4.296 and 95\% CI 2.639-6.994 for the fourth quartile). These

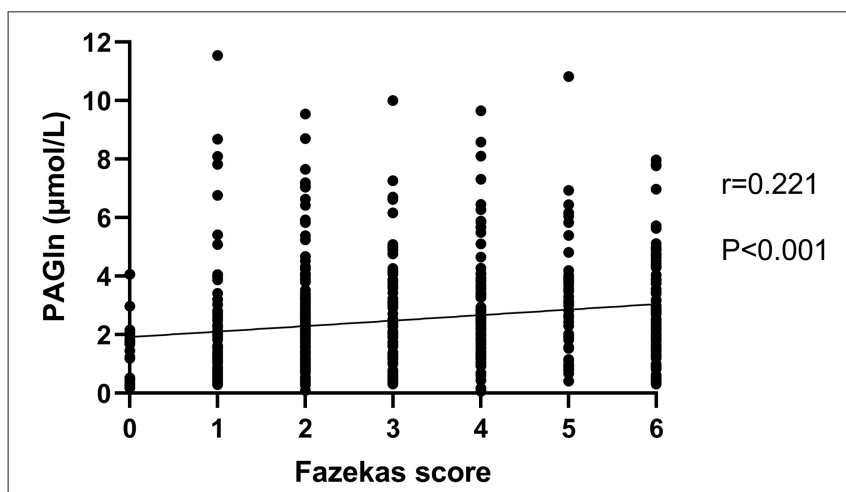

FIGURE 2 | Correlation between PAGIn levels and Fazekas score. PAGIn levels showed a significant, although limited, relationship with the total Fazekas score ( $r=0.221, P<0.001$, and Spearman rank correlation analysis). PAGln, phenylacetylglutamine. results remained significant when adjusted for age and sex. When the multivariable model was further adjusted for age, sex, hypertension, diabetes mellitus, CAD, platelet counts, eGFR, triglycerides, and homocysteine levels, only the fourth quartile of PAGIn level was independently associated with moderate-severe WMH (OR 2.134 and 95\% CI 1.134-4.018).

\section{The Association Between Plasma PAGIn and the Severity of WMH According to the Location of WMH}

To further explore the relationship between plasma PAGln and different areas of $\mathrm{WMH}$ burden, we divided all patients into a $\mathrm{P}-\mathrm{WMH}$ group and a $\mathrm{D}-\mathrm{WMH}$ group. We categorized the severity of P-WMH and D-WMH as none-mild (Fazekas score $0-1$ ) and moderate-severe (Fazekas score 2-3), respectively. There were 304 patients with none-mild P-WMH and 291 patients with moderate-severe P-WMH. Compared with patients with none-mild $\mathrm{P}-\mathrm{WMH}$, patients with moderate-severe $\mathrm{P}$ $\mathrm{WMH}$ were older and had a higher frequency of hypertension, diabetes mellitus, and CAD, higher levels of blood urea nitrogen and homocysteine, and lower levels of eGFR and triglycerides.

TABLE 3 | Logistic regression analyses of the association between PAGIn levels and overall WMH.

\begin{tabular}{|c|c|c|c|c|}
\hline & \multirow[t]{2}{*}{$P$ value } & \multirow[t]{2}{*}{ OR } & \multicolumn{2}{|c|}{$95 \% \mathrm{Cl}$ for OR } \\
\hline & & & Lower & Upper \\
\hline \multicolumn{5}{|l|}{ Unadjusted model } \\
\hline \multicolumn{5}{|l|}{ PAGIn levels } \\
\hline First quartile & Reference & & & \\
\hline Second quartile & 0.001 & 2.212 & 1.390 & 3.522 \\
\hline Third quartile & 0.061 & 1.559 & 0.980 & 2.479 \\
\hline Fourth quartile & $<0.001$ & 4.296 & 2.639 & 6.994 \\
\hline \multicolumn{5}{|l|}{ Adjusted model $^{\mathrm{a}}$} \\
\hline \multicolumn{5}{|l|}{ PAGIn levels } \\
\hline First quartile & Reference & & & \\
\hline Second quartile & 0.020 & 1.818 & 1.098 & 3.010 \\
\hline Third quartile & 0.755 & 0.921 & 0.549 & 1.544 \\
\hline Fourth quartile & 0.009 & 2.053 & 1.195 & 3.528 \\
\hline Age (years) & $<0.001$ & 1.080 & 1.059 & 1.100 \\
\hline Sex (male) & 0.781 & 0.946 & 0.642 & 1.396 \\
\hline \multicolumn{5}{|l|}{ Adjusted model ${ }^{b}$} \\
\hline \multicolumn{5}{|l|}{ PAGIn levels } \\
\hline First quartile & Reference & & & \\
\hline Second quartile & 0.085 & 1.670 & 0.932 & 2.994 \\
\hline Third quartile & 0.963 & 0.986 & 0.547 & 1.778 \\
\hline Fourth quartile & 0.019 & 2.134 & 1.134 & 4.018 \\
\hline Age (years) & $<0.001$ & 1.065 & 1.041 & 1.089 \\
\hline Sex (male vs. female) & 0.324 & 0.776 & 0.469 & 1.285 \\
\hline $\mathrm{HBP}$ & 0.399 & 1.235 & 0.757 & 2.015 \\
\hline DM & 0.804 & 1.060 & 0.668 & 1.685 \\
\hline CAD & 0.799 & 1.077 & 0.610 & 1.900 \\
\hline Platelet $>204 \times 10^{9} / L$ & 0.195 & 0.755 & 0.494 & 1.155 \\
\hline eGFR $\leq 83.85 \mathrm{~mL} / \mathrm{min} / 1.73 \mathrm{~m}^{2}$ & 0.125 & 1.421 & 0.908 & 2.224 \\
\hline $\mathrm{TG}>1.52 \mathrm{mmol} / \mathrm{L}$ & 0.262 & 0.785 & 0.514 & 1.198 \\
\hline Homocysteine $>13.28 \mu \mathrm{mol} / \mathrm{L}$ & 0.181 & 1.359 & 0.867 & 2.129 \\
\hline
\end{tabular}

Adjusted model' : adjusted for age and sex.

Adjusted model ${ }^{b}$ : adjusted for age, sex, HBP, DM, CAD, platelet counts, eGFR, TG, and homocysteine levels.

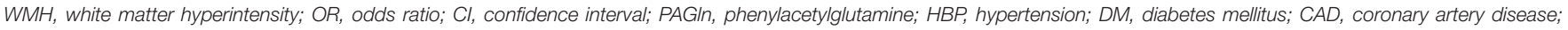
eGFR, estimated glomerular filtration rate; TG, triglycerides. 
When classified by D-WMH, 348 and 247 patients were in the none-mild and moderate-severe D-WMH groups, respectively. Patients with moderate-severe $\mathrm{D}$-WMH were more likely to have hypertension and CAD, higher systolic blood pressure, higher levels of blood urea nitrogen, high-density lipoprotein, and homocysteine, and lower levels of eGFR (Table 4).

Levels of PAGln in the P-WMH and D-WMH groups are shown in Figures 1B,C. PAGln levels were elevated in patients with moderate-severe WMH. Binary logistic regression analyses demonstrated that the fourth PAGln quartile was independently associated with severe P-WMH (OR 2.227 and 95\% CI 1.1744.226) (using the first quartile as the reference) when adjusted for age, sex, hypertension, diabetes mellitus, CAD, blood urea nitrogen, eGFR, triglycerides, and homocysteine levels. However, the significant association between the second and fourth quartiles of PAGln levels with severe D-WMH disappeared when adjustments were made for age, sex, vascular risk factors, and laboratory biomarkers (Table 5).

\section{Receiver Operating Characteristic Analyses of PAGIn Levels According to the Severity of WMH}

The diagnostic value of PAGln in distinguishing ischemic stroke patients according to $\mathrm{WMH}$ burden was evaluated using the ROC analysis. The AUCs for overall WMH, P-WMH, and D-WMH were $0.616,0.635$, and 0.579 (Figure 3), respectively. The optimal PAGln cut-off values were $3.348,3.075$, and $3.341 \mu \mathrm{mol} / \mathrm{L}$ for overall $\mathrm{WMH}, \mathrm{P}-\mathrm{WMH}$, and $\mathrm{D}-\mathrm{WMH}$, respectively.

\section{DISCUSSION}

In this study, we conducted a targeted metabolomic analysis to explore the association between PAGln levels and WMH in patients with ischemic stroke. Our results demonstrated that plasma PAGln levels at admission were associated with the severity of WMH in patients with ischemic stroke. After adjusting for age, sex, and confounding factors, higher PAGln levels were independently associated with moderate-severe overall WMH. These associations were also found with P-WMH but not with D-WMH.

The pathophysiology of WMH remains unclear. Traditional vascular risk factors such as age, hypertension, diabetes mellitus, and smoking may play crucial roles in the pathological process of WMH and SVD (Rost et al., 2010; Giese et al., 2020). Previous studies have uncovered biomarkers of endothelial dysfunction, inflammation, and impaired fibrinolysis for $\mathrm{WMH}$ in stroke patients and the general population (Poggesi et al., 2016). Metabolomic biomarkers such as uric acid, homocysteine,

TABLE 4 | Characteristics of patients according to the scales of P-WMH and D-WMH.

\begin{tabular}{|c|c|c|c|c|c|c|}
\hline \multirow[t]{2}{*}{ Variables } & \multicolumn{3}{|c|}{ P-WMH } & \multicolumn{3}{|c|}{ D-WMH } \\
\hline & $\begin{array}{c}\text { None-mild } \\
n=304\end{array}$ & $\begin{array}{c}\text { Moderate-severe } \\
\qquad n=291\end{array}$ & $P$ value & $\begin{array}{c}\text { None-mild } \\
n=348\end{array}$ & $\begin{array}{c}\text { Moderate-severe } \\
\qquad n=247\end{array}$ & $P$ value \\
\hline Age (years) & $55.0(49.0-63.0)$ & $67.0(60.0-72.0)$ & $<0.001$ & $57.0(50.0-64.0)$ & 67.0 (59.0-72.0) & $<0.001$ \\
\hline Sex (male, $N, \%$ ) & 87 (28.6\%) & 105 (36.1\%) & 0.052 & 244 (70.1\%) & 159 (64.4\%) & 0.140 \\
\hline $\operatorname{HBP}(N, \%)$ & $191(62.8 \%)$ & 233 (80.1\%) & $<0.001$ & 223 (64.1\%) & 201 (81.4\%) & $<0.001$ \\
\hline $\mathrm{DM}(N, \%)$ & 73 (24.0\%) & 99 (34.0\%) & 0.007 & $98(28.2 \%)$ & 74 (30.0\%) & 0.633 \\
\hline Hyperlipidemia (N, \%) & 88 (28.9\%) & 85 (29.2\%) & 0.944 & 95 (27.3\%) & 78 (31.6\%) & 0.257 \\
\hline CAD (N, \%) & 37 (12.2\%) & 65 (22.3\%) & 0.001 & 44 (12.6\%) & 58 (23.5\%) & $<0.001$ \\
\hline Smoking (N, \%) & 153 (50.3\%) & 131 (45.0\%) & 0.195 & 169 (48.6\%) & 115 (46.6\%) & 0.630 \\
\hline Drinking (N, \%) & 108 (35.5\%) & 105 (36.1\%) & 0.888 & 124 (35.6\%) & 89 (36.0\%) & 0.920 \\
\hline $\mathrm{SBP}(\mathrm{mmHg})$ & $142.0(127.0-155.0)$ & $143.0(130.0-158.5)$ & 0.096 & $141.0(129.0-154.0)$ & $145.0(130.0-161.0)$ & 0.017 \\
\hline DBP (mmHg) & 83.0 (74.0-94.0) & 82.0 (73.0-90.0) & 0.190 & $83.0(74.0-92.0)$ & $82.0(73.0-91.5)$ & 0.613 \\
\hline BMl & 23.6 (22.0-25.2) & $23.0(20.8-25.8)$ & 0.279 & 23.3 (21.9-25.6) & 23.4 (20.9-25.7) & 0.720 \\
\hline PAGln ( $\mu \mathrm{mmol} / \mathrm{L})$ & $1.8(1.0-2.7)$ & $2.4(1.5-3.8)$ & $<0.001$ & $1.9(1.1-2.9)$ & $2.4(1.5-3.8)$ & $<0.001$ \\
\hline White blood cell count $\left(\times 10^{9} / \mathrm{L}\right)$ & $6.7(5.4-8.2)$ & $6.7(5.6-8.0)$ & 0.884 & $6.7(5.5-8.1)$ & $6.7(5.5-8.0)$ & 0.904 \\
\hline Platelet $\left(\times 10^{9} / L\right)$ & $207.0(167.8-249.0)$ & 199.0 (163.0-239.5) & 0.113 & 207.0 (167.8-249.0) & 199.0 (163.0-239.5) & 0.113 \\
\hline BUN (mmol/L) & $4.9(3.9-5.9)$ & $5.2(4.2-6.4)$ & 0.009 & $5.0(3.9-6.0)$ & $5.1(4.2-6.3)$ & 0.033 \\
\hline eGFR $\left(\mathrm{ml} / \mathrm{min} / 1.73 \mathrm{~m}^{2}\right)$ & $88.5(74.0-98.7)$ & $77.7(64.2-89.1)$ & $<0.001$ & 88.4 (74.6-98.2) & 76.7 (62.2-88.2) & $<0.001$ \\
\hline Uric acid ( $\mu \mathrm{mol} / \mathrm{L})$ & 318.6 (273.1-385.7) & $328.1(267.1-385.8)$ & 0.799 & $315.0(272.3-379.1)$ & $339.8(271.2-393.2)$ & 0.204 \\
\hline TC (mmol/L) & $4.2(3.4-5.2)$ & $4.2(3.4-4.9)$ & 0.143 & $4.2(3.4-5.2)$ & $4.3(3.5-5.0)$ & 0.415 \\
\hline TG (mmol/L) & $1.6(1.2-2.2)$ & $1.5(1.0-2.2)$ & 0.045 & $1.6(1.1-2.2)$ & $1.5(1.0-2.3)$ & 0.154 \\
\hline HDL (mmol/L) & $1.0(0.8-1.2)$ & $1.0(0.9-1.2)$ & 0.669 & $1.0(0.8-1.1)$ & $1.0(0.9-1.2)$ & 0.004 \\
\hline LDL (mmol/L) & $2.6(2.1-3.4)$ & $2.6(2.0-3.1)$ & 0.123 & $2.6(2.0-3.3)$ & $2.6(2.1-3.2)$ & 0.565 \\
\hline Fasting blood-glucose (mmol/L) & $5.6(4.9-6.9)$ & $5.7(5.0-7.7)$ & 0.213 & $5.6(5.0-7.4)$ & $5.7(5.0-7.3)$ & 0.882 \\
\hline $\mathrm{HbA1c}(\%)$ & $5.8(5.4-6.5)$ & $6.0(5.5-7.1)$ & 0.314 & $5.8(5.4-7.2)$ & $5.9(5.6-6.8)$ & 0.647 \\
\hline Homocysteine ( $\mu \mathrm{mol} / \mathrm{L})$ & $12.7(10.8-15.1)$ & $14.0(11.4-17.8)$ & $<0.001$ & $12.8(10.8-15.5)$ & $14.0(11.6-18.3)$ & 0.001 \\
\hline
\end{tabular}

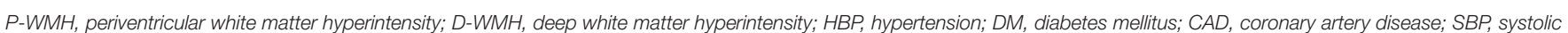

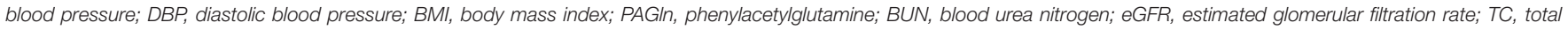
cholesterol; TG, triglycerides; HDL, high-density lipoprotein; LDL, low-density lipoprotein; HbA1c, glycosylated hemoglobin A1c. 
TABLE 5 | Logistic regression analyses of the association between PAGIn levels and P-WMH and D-WMH.

\begin{tabular}{|c|c|c|c|c|c|c|c|c|c|}
\hline & \multicolumn{4}{|c|}{ P-WMH } & & \multicolumn{4}{|c|}{ D-WMH } \\
\hline & \multirow[t]{2}{*}{$P$ value } & \multirow[t]{2}{*}{ OR } & \multicolumn{2}{|c|}{$95 \% \mathrm{Cl}$ for OR } & & \multirow[t]{2}{*}{$P$ value } & \multirow[t]{2}{*}{ OR } & \multicolumn{2}{|c|}{$95 \% \mathrm{Cl}$ for OR } \\
\hline & & & Lower & Upper & & & & Lower & Upper \\
\hline Unadjusted model & & & & & Unadjusted model & & & & \\
\hline PAGln levels & & & & & PAGIn levels & & & & \\
\hline First quartile & Reference & & & & First quartile & Reference & & & \\
\hline Second quartile & 0.003 & 2.021 & 1.263 & 3.235 & Second quartile & 0.022 & 1.748 & 1.083 & 2.823 \\
\hline Third quartile & 0.024 & 1.719 & 1.073 & 2.755 & Third quartile & 0.177 & 1.396 & 0.860 & 2.266 \\
\hline Fourth quartile & $<0.001$ & 4.816 & 2.9491 & 7.867 & Fourth quartile & $<0.001$ & 3.220 & 1.993 & 5.201 \\
\hline Adjusted model ${ }^{\mathrm{a}}$ & & & & & Adjusted model ${ }^{\mathrm{a}}$ & & & & \\
\hline PAGIn levels & & & & & PAGIn levels & & & & \\
\hline First quartile & Reference & & & & First quartile & Reference & & & \\
\hline Second quartile & 0.062 & 1.637 & 0.976 & 2.747 & Second quartile & 0.171 & 1.428 & 0.858 & 2.379 \\
\hline Third quartile & 0.983 & 0.994 & 0.585 & 1.689 & Third quartile & 0.629 & 0.878 & 0.518 & 1.488 \\
\hline Fourth quartile & 0.004 & 2.247 & 1.297 & 3.890 & Fourth quartile & 0.065 & 1.648 & 0.970 & 2.799 \\
\hline Age (years) & $<0.001$ & 1.088 & 1.067 & 1.110 & Age (years) & $<0.001$ & 1.068 & 1.049 & 1.088 \\
\hline Sex (male) & 0.494 & 0.872 & 0.588 & 1.291 & Sex (male) & 0.684 & 0.924 & 0.633 & 1.349 \\
\hline Adjusted model ${ }^{b}$ & & & & & Adjusted model ${ }^{b}$ & & & & \\
\hline PAGln levels & & & & & PAGIn levels & & & & \\
\hline First quartile & Reference & & & & First quartile & Reference & & & \\
\hline Second quartile & 0.286 & 1.383 & 0.762 & 2.511 & Second quartile & 0.475 & 1.243 & 0.685 & 2.255 \\
\hline Third quartile & 0.859 & 1.056 & 0.577 & 1.933 & Third quartile & 0.730 & 0.899 & 0.489 & 1.651 \\
\hline Fourth quartile & 0.014 & 2.227 & 1.174 & 4.226 & Fourth quartile & 0.057 & 1.819 & 0.981 & 3.372 \\
\hline Age (years) & $<0.001$ & 1.077 & 1.051 & 1.103 & Age (years) & $<0.001$ & 1.054 & 1.031 & 1.078 \\
\hline Sex (male vs. female) & 0.377 & 0.796 & 0.480 & 1.321 & Sex (male vs. female) & 0.750 & 1.082 & 0.665 & 1.761 \\
\hline HBP & 0.377 & 1.255 & 0.759 & 2.075 & $\mathrm{HBP}$ & 0.076 & 1.609 & 0.951 & 2.723 \\
\hline DM & 0.414 & 1.215 & 0.761 & 1.939 & CAD & 0.724 & 1.103 & 0.640 & 1.901 \\
\hline CAD & 0.974 & 0.990 & 0.561 & 1.749 & $\mathrm{SBP}>142 \mathrm{mmHg}$ & 0.692 & 1.092 & 0.708 & 1.684 \\
\hline $\mathrm{BUN}>5.01 \mathrm{mmol} / \mathrm{L}$ & 0.899 & 0.972 & 0.623 & 1.515 & $\mathrm{BUN}>5.01 \mathrm{mmol} / \mathrm{L}$ & 0.307 & 0.795 & 0.512 & 1.235 \\
\hline $\mathrm{eGFR} \leq 83.85 \mathrm{~mL} / \mathrm{min} / 1.73 \mathrm{~m}^{2}$ & 0.305 & 1.277 & 0.800 & 2.038 & $\mathrm{eGFR} \leq 83.85 \mathrm{~mL} / \mathrm{min} / 1.73 \mathrm{~m}^{2}$ & 0.051 & 1.584 & 0.998 & 2.513 \\
\hline $\mathrm{TG}>1.52 \mathrm{mmol} / \mathrm{L}$ & 0.154 & 0.733 & 0.479 & 1.123 & $\mathrm{HDL}>1.00 \mathrm{mmol} / \mathrm{L}$ & 0.006 & 1.847 & 1.196 & 2.853 \\
\hline Homocysteine $>13.28 \mu \mathrm{mol} / \mathrm{L}$ & 0.119 & 1.437 & 0.910 & 2.268 & Homocysteine $>13.28 \mu \mathrm{mol} / \mathrm{L}$ & 0.260 & 1.303 & 0.822 & 2.067 \\
\hline
\end{tabular}

Adjusted modela: adjusted for age and sex.

P-WMH adjusted model ${ }^{b}$ : adjusted for age, sex, HBP, DM, CAD, BUN, eGFR, TG, and homocysteine levels.

$D-W M H$ adjusted model ${ }^{b}$ : adjusted for age, sex, HBP, CAD, SBP, BUN, eGFR, HDL, and homocysteine levels.

WMH, white matter hyperintensity; P-WMH, periventricular white matter hyperintensity; D-WMH, deep white matter hyperintensity; OR, odds ratio; Cl, confidence interval; PAGln,

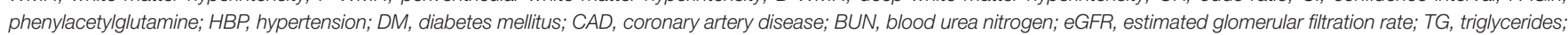
HDL, high-density lipoprotein; SBP, systolic blood pressure.

AMDA, and ceramides have been reported to be related to WMH (Han et al., 2016). Of note, these altered metabolites might be involved in the pathological process of $\mathrm{WMH}$ through their common role in endothelial dysfunction. Many studies have investigated the role of microbiota in neurological disorders, but studies on WMH and SVD are relatively rare. Recently, Cai et al. reported on the role of the gutimmune-brain axis in arteriosclerotic SVD pathophysiology (Cai et al., 2021). Another cross-sectional study indicated that some microbiota may increase the risk of WMH and SVD (Saji et al., 2021).

Gut microbiota can produce metabolites or toxins that influence the health of the host. Gut microbiota-derived metabolites, such as trimethylamine- $N$-oxide (TMAO), tryptophan, and indole derivatives, may play critical roles in the pathogenesis of cardiovascular and cerebrovascular diseases (Ascher and Reinhardt, 2018; Wang and Zhao, 2018). TMAO has been the most studied gut microbiota-derived metabolite in recent years. Accumulating evidence has proven the causal links among TMAO, CAD, and stroke (Witkowski et al., 2020). Elevated TMAO and choline levels have recently been found to be associated with severe WMHs, especially P-WMH (Chen et al., 2021).

Phenylacetylglutamine, another gut microbial metabolite, has been reported to correlate with chronic kidney disease, diabetes mellitus, cardiovascular disease, and Parkinson's disease (Poesen et al., 2016; Urpi-Sarda et al., 2019; Shao et al., 2021). In 2020, Hazen et al. identified a causal contribution of PAGln to incident 

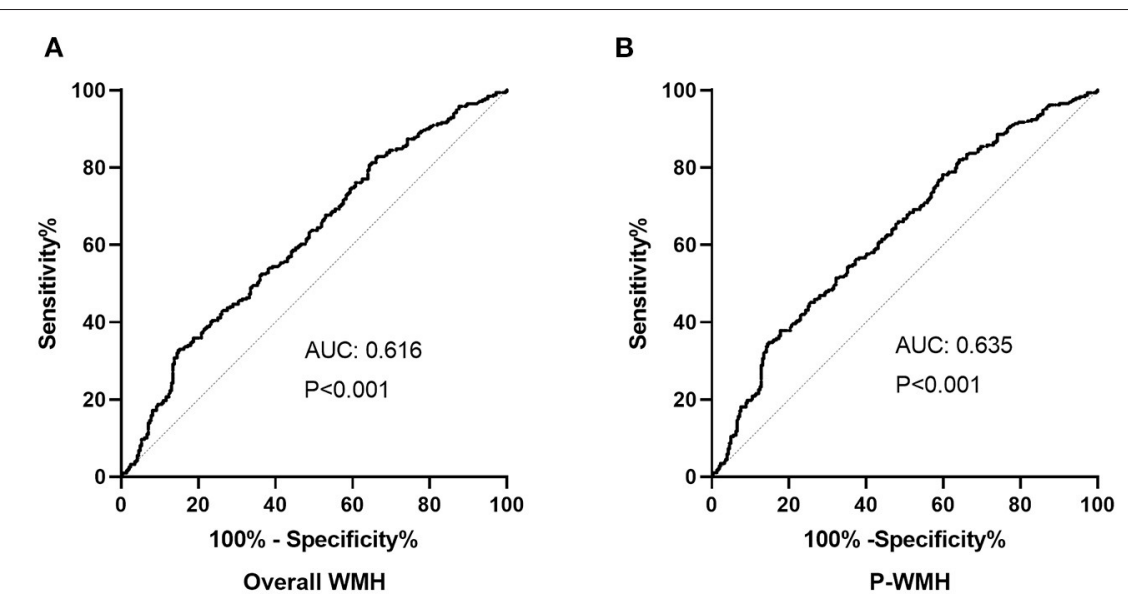

C

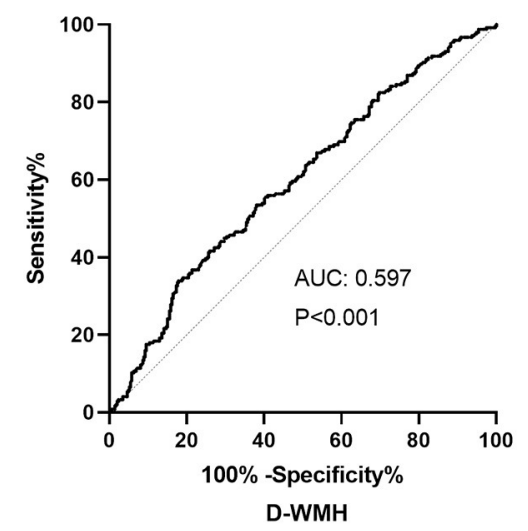

FIGURE 3 | Receiver operating characteristic analysis of PAGln according to the severity of WMH. (A) The AUC was 0.616, and the optimal PAGIn level cut-off value was $3.348 \mu \mathrm{mol} / \mathrm{L}$ for overall WMH. (B) The AUC was 0.635, and the optimal PAGln level cut-off value was 3.075 $\mu \mathrm{mol} / \mathrm{L}$ for $\mathrm{P}-\mathrm{WMH}$. (C) The AUC was 0.597, and the optimal PAGIn level cut-off value was $3.341 \mu \mathrm{mol} / \mathrm{L}$ for $\mathrm{D}-\mathrm{WMH}$. ROC, receiver operating characteristic curve; PAGln, phenylacetylglutamine; WMH, white matter hyperintensity; P-WMH, periventricular white matter hyperintensity; D-WMH, deep white matter hyperintensity; AUC, area under the curve.

cardiovascular disease risks in a large sample clinical study (Nemet et al., 2020). This study suggested a clinical association between elevated PAGln levels and the overall burden of WMH and P-WMH. The possible mechanisms are as follows: first, studies have shown that PAGln levels are positively associated with age (Swann et al., 2013; Poesen et al., 2016), and our data also showed an increase in PAGln levels with increasing age, which is a known factor contributing to the pathology of WMH (Urpi-Sarda et al., 2019). Second, traditional vascular risk factors, such as hypertension and diabetes mellitus, are involved in the process of WMH (Tamura and Araki, 2015). As shown in Table 1, the group of patients with higher PAGln levels had higher rates of hypertension and diabetes, and studies have also suggested higher PAGln levels in patients with diabetes (Nemet et al., 2020), and therefore the relationship between PAGln and WMH might be due to the underlying mechanism of small vessel abnormalities of hypertension and diabetes (Tamura and Araki, 2015). Our data showed a decrease in eGFR with increasing PAGln levels, and previous observations also showed that kidney impairment measured by eGFR was strongly associated with high serum PAGln levels (Wang and Zhao, 2018). Furthermore, we found that decreased eGFR was associated with moderate-severe $\mathrm{WMH}$, consistent with the previous results (Steinicke et al., 2012; Zong et al., 2016).

In this study, WMH was divided into $\mathrm{P}-\mathrm{WMH}$ and $\mathrm{D}-\mathrm{WMH}$. A limited number of studies have investigated the differences between $\mathrm{P}-\mathrm{WMH}$ and $\mathrm{D}-\mathrm{WMH}$; however, the underlying mechanism has not yet been fully elucidated. Our results suggest the involvement of PAGln in the development of P-WMH, but not $\mathrm{D}-\mathrm{WMH}$, and the detailed mechanisms require further investigation. Previous pathology studies have shown that P$\mathrm{WMH}$ is more likely to be associated with inflammation and chronic hypoperfusion, whereas D-WMH is related to ischemic damage (Fazekas et al., 1993). These differences may provide possible explanations for the relationship between PAGln and
P-WMH. Previous studies have found a relationship between PAGln levels and human immunodeficiency virus-associated dementia and impaired cognitive function in patients receiving hemodialysis (Cassol et al., 2014; Kurella Tamura et al., 2016). As a uremic metabolite, PAGln can lead to blood-brain barrier disruption and impair P-WMH. In addition, there are some controversies regarding the relationship between diabetes and $\mathrm{P}-\mathrm{WMH}$ and $\mathrm{D}-\mathrm{WMH}$. In our data, we found a higher rate of diabetes in patients with moderate-severe P-WMH; however, no difference was found in patients with D-WMH. Limited studies (Urpi-Sarda et al., 2019; Nemet et al., 2020) have revealed the associations between diabetes and PAGln levels. The closer relationship between diabetes and P-WMH might be the reason why PAGln is associated more with P-WMH than D-WMH.

There were some limitations to this study. First, this was a cross-sectional study, so we could not establish a causal relationship between PAGln and WMH. Second, participants in our study were recruited from a single center, and this could have led to patient selection bias. Third, PAGln levels were only analyzed at a single time point, and information on dynamic changes in PAGln was missing. Fourth, investigations of the gut microbiota were lacking in this study. Finally, we used a less precise visual rating scale to assess the degree of WMH. Quantification of WMH is needed to further investigate the relationship between PAGln and WMH volume.

\section{CONCLUSION}

In conclusion, higher plasma PAGln levels might be a biomarker of moderate-severe WMH, especially moderate-severe P-WMH. Further studies concerning the cause-effect relationship between PAGln and WMH are needed. 


\section{DATA AVAILABILITY STATEMENT}

The datasets generated for this study are available on request to the corresponding author.

\section{ETHICS STATEMENT}

The studies involving human participants were reviewed and approved by Xiangya Hospital Ethics Committee. The patients/participants provided their written informed consent to participate in this study.

\section{AUTHOR CONTRIBUTIONS}

FY and XF: methodology and writing-original draft preparation. XL, YL, MW, and TZ: investigation and data curation. JX: conceptualization and writing-reviewing and editing. All authors contributed to the article and approved the submitted version.

\section{REFERENCES}

Arsava, E. M., Rahman, R., Rosand, J., Lu, J., Smith, E. E., Rost, N. S., et al. (2009). Severity of leukoaraiosis correlates with clinical outcome after ischemic stroke. Neurology 72, 1403-1410. doi: 10.1212/WNL.0b013e3181al 8823

Ascher, S., and Reinhardt, C. (2018). The gut microbiota: an emerging risk factor for cardiovascular and cerebrovascular disease. Eur. J. Immunol. 48, 564-575. doi: 10.1002/eji.201646879

Cai, W., Chen, X., Men, X., Ruan, H., Hu, M., Liu, S., et al. (2021). Gut microbiota from patients with arteriosclerotic CSVD induces higher IL17A production in neutrophils via activating ROR $\gamma$ t. Sci. Adv. 7: eabe4827. doi: 10.1126/sciadv.abe4827

Cassol, E., Misra, V., Dutta, A., Morgello, S., and Gabuzda, D. (2014). Cerebrospinal fluid metabolomics reveals altered waste clearance and accelerated aging in HIV patients with neurocognitive impairment. AIDS 28, 1579-1591. doi: 10.1097/QAD.0000000000000303

Chen, Y., Xu, J., Pan, Y., Yan, H., Jing, J., Yang, Y., et al. (2021). Association of trimethylamine $\mathrm{N}$-oxide and its precursor with cerebral small vessel imaging markers. Front. Neurol. 12:648702. doi: 10.3389/fneur.2021.648702

Fazekas, F., Kleinert, R., Offenbacher, H., Schmidt, R., Kleinert, G., Payer, F., et al. (1993). Pathologic correlates of incidental MRI white matter signal hyperintensities. Neurology 43, 1683-1689. doi: 10.1212/WNL.43.9.1683

Feng, X., Yu, F., Zhou, X., Liu, Z., Liao, D., Huang, Q., et al. (2021). MMP9 rs17576 Is simultaneously correlated with symptomatic intracranial atherosclerotic stenosis and white matter hyperintensities in Chinese population. Cerebrovasc. Dis. 50, 4-11. doi: 10.1159/000511582

Giese, A. K., Schirmer, M. D., Dalca, A. V., Sridharan, R., Donahue, K. L., Nardin, M., et al. (2020). White matter hyperintensity burden in acute stroke patients differs by ischemic stroke subtype. Neurology 95, e79-e88. doi: 10.1212/WNL.0000000000009728

Han, S. W., Song, T. J., Bushnell, C. D., Lee, S. S., Kim, S. H., Lee, J. H., et al. (2016). Serum uric acid is associated with cerebral white matter hyperintensities in patients with acute lacunar infarction. J. Neuroimaging 26, 351-354. doi: $10.1111 /$ jon. 12308

Janes, F., Cif,ù, A., Pessa, M. E., Domenis, R., Gigli, G. L., Sanvilli, N., et al. (2019). ADMA as a possible marker of endothelial damage. A study in young asymptomatic patients with cerebral small vessel disease. Sci. Rep. 9:14207. doi: 10.1038/s41598-019-50778-w

Joutel, A., and Chabriat, H. (2017). Pathogenesis of white matter changes in cerebral small vessel diseases: beyond vessel-intrinsic mechanisms. Clin. Sci. 131, 635-651. doi: 10.1042/CS20160380

\section{FUNDING}

This study was supported by the Project Program of National Clinical Research Center for Geriatric Disorders (Xiangya Hospital, Grant No. 2020LNJJ16), the National Natural Science Foundation of China (Grant No. 81671166), the Fundamental Research Funds for the Central Universities of Central South University (Grant No. 2019zzts902), and the Provincial Key Plan for Research and Development of Hunan (Grant No. 2020SK2067).

\section{ACKNOWLEDGMENTS}

We thank Zhimin Long, Haihong Zha, and Mengqin Xiao from SCIEX, Analytical Instrument Trading Co., Ltd, Shanghai, China for their help in sample testing and analysis of metabolomics data. We also thank Editage (www.editage.cn) for their linguistic assistance during the revision of this manuscript.

Kurella Tamura, M., Chertow, G. M., Depner, T. A., Nissenson, A. R., Schiller, B., Mehta, R. L., et al. (2016). Metabolic profiling of impaired cognitive function in patients receiving dialysis. J. Am. Soc. Nephrol. 27, 3780-3787. doi: 10.1681/ASN.2016010039

Moldave, K., and Meister, A. (1957). Synthesis of phenylacetylglutamine by human tissue. J. Biol. Chem. 229, 463-476. doi: 10.1016/S0021-9258(18)70632-7

Nemet, I., Saha, P. P., Gupta, N., Zhu, W., Romano, K. A., Skye, S. M., et al. (2020). A cardiovascular disease-linked gut microbial metabolite acts via adrenergic receptors. Cell 180, 862-877.e822. doi: 10.1016/j.cell.2020.02.016

Nie, J., Xie, L., Zhao, B. X., Li, Y., Qiu, B., Zhu, F., et al. (2018). Serum trimethylamine $\mathrm{N}$-oxide concentration is positively associated with first stroke in hypertensive patients. Stroke 49, 2021-2028. doi: 10.1161/STROKEAHA.118.021997

Park, J. H., Heo, S. H., Lee, M. H., Kwon, H. S., Kwon, S. U., and Lee, J. S. (2019). White matter hyperintensities and recurrent stroke risk in patients with stroke with small-vessel disease. Eur. J. Neurol. 26, 911-918. doi: 10.1111/ene.13908

Poesen, R., Claes, K., Evenepoel, P., de Loor, H., Augustijns, P., Kuypers, D., et al. (2016). Microbiota-derived phenylacetylglutamine associates with overall mortality and cardiovascular disease in patients with CKD. J. Am. Soc. Nephrol. 27, 3479-3487. doi: 10.1681/ASN.2015121302

Poggesi, A., Pasi, M., Pescini, F., Pantoni, L., and Inzitari, D. (2016). Circulating biologic markers of endothelial dysfunction in cerebral small vessel disease: a review. J. Cereb. Blood Flow Metab. 36, 72-94. doi: 10.1038/jcbfm.2015.116

Rist, P. M., Buring, J. E., Rexrode, K. M., Cook, N. R., and Rost, N. S. (2019). Prospectively collected lifestyle and health information as risk factors for white matter hyperintensity volume in stroke patients. Eur. J. Epidemiol. 34, 957-965. doi: 10.1007/s10654-019-00546-x

Rost, N. S., Rahman, R., Sonni, S., Kanakis, A., Butler, C., Massasa, E., et al. (2010). Determinants of white matter hyperintensity volume in patients with acute ischemic stroke. J. Stroke Cerebrovasc. Dis. 19, 230-235. doi: 10.1016/j.jstrokecerebrovasdis.2009.05.007

Saji, N., Murotani, K., Hisada, T., Tsuduki, T., Sugimoto, T., Kimura, A., et al. (2021). The association between cerebral small vessel disease and the gut microbiome: a cross-sectional analysis. J. Stroke Cerebrovasc. Dis. 30, 105568. doi: 10.1016/j.jstrokecerebrovasdis.2020.105568

Shao, Y., Li, T., Liu, Z., Wang, X., Xu, X., Li, S., et al. (2021). Comprehensive metabolic profiling of Parkinson's disease by liquid chromatography-mass spectrometry. Mol. Neurodegener. 16:4. doi: 10.1186/s13024-021-00425-8

Steinicke, R., Gaertner, B., Grittner, U., Schmidt, W., Dichgans, M., Heuschmann, P. U., et al. (2012). Kidney function and white matter disease in young stroke patients: analysis of the stroke in young fabry patients study population. Stroke 43, 2382-2388. doi: 10.1161/STROKEAHA.111.645713 
Swann, J. R., Spagou, K., Lewis, M., Nicholson, J. K., Glei, D. A., Seeman, T. E., et al. (2013). Microbial-mammalian cometabolites dominate the age-associated urinary metabolic phenotype in Taiwanese and American populations. J. Proteome Res. 12, 3166-3180. doi: 10.1021/pr4000152

Tamura, Y., and Araki, A. (2015). Diabetes mellitus and white matter hyperintensity. Geriatr. Gerontol. Int. 15 (Suppl 1), 34-42. doi: 10.1111 /ggi.12666

Urpi-Sarda, M., Almanza-Aguilera, E., Llorach, R., Vázquez-Fresno, R., Estruch, R., Corella, D., et al. (2019). Non-targeted metabolomic biomarkers and metabotypes of type 2 diabetes: a cross-sectional study of PREDIMED trial participants. Diabetes Metab. 45, 167-174. doi: 10.1016/j.diabet.2018.02.006

Wang, Z., and Zhao, Y. (2018). Gut microbiota derived metabolites in cardiovascular health and disease. Protein Cell 9, 416-431. doi: 10.1007/s13238-018-0549-0

Witkowski, M., Weeks, T. L., and Hazen, S. L. (2020). Gut microbiota and cardiovascular disease. Circ. Res. 127, 553-570. doi: 10.1161/CIRCRESAHA.120. 316242

Wu, S., Wu, B., Liu, M., Chen, Z., Wang, W., Anderson, C. S., et al. (2019). Stroke in China: advances and challenges in epidemiology, prevention, and management. Lancet Neurol. 18, 394-405. doi: 10.1016/S1474-4422(18) 30500-3

Yu, L., Yang, L., Zhang, X., Yuan, J., Li, Y., Yang, S., et al. (2018). Age and recurrent stroke are related to the severity of white matter hyperintensities in lacunar infarction patients with diabetes. Clin. Interv. Aging 13, 2487-2494. doi: 10.2147/CIA. S184463
Zhu, S., Qian, S., Xu, T., Peng, H., Dong, R., Wang, D., et al. (2020). White matter hyperintensity, immediate antihypertensive treatment, and functional outcome after acute ischemic stroke. Stroke 51, 1608-1612. doi: 10.1161/STROKEAHA.119.028841

Zong, L., Yao, M., Ni, J., Zhou, L., Yuan, J., Peng, B., et al. (2016). Kidney function is associated with severity of white matter hyperintensity in patients with acute ischemic stroke/TIA. BMC Neurol. 16, 193. doi: 10.1186/s12883-0160714-0

Conflict of Interest: The authors declare that the research was conducted in the absence of any commercial or financial relationships that could be construed as a potential conflict of interest.

Publisher's Note: All claims expressed in this article are solely those of the authors and do not necessarily represent those of their affiliated organizations, or those of the publisher, the editors and the reviewers. Any product that may be evaluated in this article, or claim that may be made by its manufacturer, is not guaranteed or endorsed by the publisher.

Copyright $\odot 2021 \mathrm{Yu}$, Feng, Li, Luo, Wei, Zhao and Xia. This is an open-access article distributed under the terms of the Creative Commons Attribution License (CC $B Y)$. The use, distribution or reproduction in other forums is permitted, provided the original author(s) and the copyright owner(s) are credited and that the original publication in this journal is cited, in accordance with accepted academic practice. No use, distribution or reproduction is permitted which does not comply with these terms. 